\title{
Nach dem Streik ist vor dem Streik
}

\section{Einleitung in den Schwerpunkt}

Für die Aufwertung der Sozial- und Erziehungsberufe (SuE) sind fast alle Beteiligten, nicht nur das betroffene Personal und die Fachwissenschaft : Vor allem bezogen auf die Kindertagesstätten sorgen sich auch die Politiker und Arbeitgeber mit breiter medialer Resonanz spätestens seit der Diskussion über die notwendigen Konsequenzen aus den PISA-Studien um die Qualität der frühkindlichen Bildung. Dies sei eine wesentliche Grundlage für die Zukunft Deutschlands im internationalen Wettbewerb um die „besten Köpfe“.

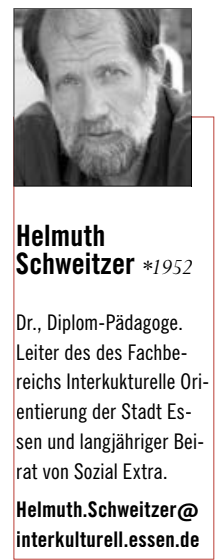

Der Widerspruch zwischen diesem theoretisch unstrittigen Anspruch mit der daraus abgeleiteten diesjährigen Zehn-Prozent-Forderung einerseits und der (trotz vierwöchigen Streiks) nicht erreichten Umsetzung andererseits ist offensichtlich: Was haben die bisherigen Tarifkonflikte für das direkt betroffene Personal, die Gewerkschaften und die Arbeitgeber gebracht? Aus Sicht des enttäuschten Kita-Personals dauert es noch Jahre, bis der Verdienstausfall angesichts des niedrigeren Streikgeldes durch die Verbesserungen in der Eingruppierung ausgeglichen ist. Die Kommunalen Arbeitgeber haben vier Wochen lang Lohnkosten in dreistelliger Millionenhöhe gespart.

Der mit zunehmender Dauer auch gegen die Medien (als einflussreiches Sprachrohr „empörter“ Eltern) geführte und mit wachsender Mobilisierung schließlich für die Gewerkschaftskassen kostenträchtige Arbeitskampf hat vor allem die Streikenden kurzfristig mobilisiert und zunächst die Tarifpartner überrascht, ist aber inzwischen im Bewusstsein der meisten Akteure an der Basis abgehakt: Mit noch streikbereiten ErzieherInnen im Rücken wurde der Ver.di -Vorsitzende Frank Bsirske Mitte September wiedergewählt. Durch den nur acht Tage später vorgelegten Verhandlungskompromiss haben die Arbeitgeber das sechs Wochen zuvor noch von den Gewerkschaftsmitgliedern zu 69 Prozent abgelehnte Schlichtungsergebnis im Volumen nur minimal ausweiten müssen. Die Tarifpartner haben es dann soweit umverteilt, dass der Frust der ErzieherInnen und SozialarbeiterInnen angesichts der mageren Resultate von durchschnittlich zwei bis vier Prozent mehr erst mal auffangen werden konnte.

Die Beiträge dieses Schwerpunkts beleuchten den Verlauf, das Ergebnis und die Lehren des Arbeitskampfes für die bereits angekündigte Fortsetzung in den nächsten Jahren aus verschiedenen Blickwinkeln. Dabei kommen nicht nur die unterschiedlichen Strategien und Kräfteverhältnisse zwischen ver.di und GEW zum Ausdruck ${ }^{2}$. Der Umstand, dass sich einige ver.di-interne KritikerInnen an der Basis und im Gewerkschaftsapparat nur anonym äußern, zeigt auch, welches interne Konfliktpotential die zwiespältigen Erfahrungen während des Tarifkonflikts für kommende Auseinandersetzungen in sich bergen.
Ralf Haderlein begründet mit Blick auf die neuen akademischen Ausbildungsgänge zu staatlich anerkannten KindheitspädagogInnen, warum aus fachwissenschaftlicher Sicht pädagogische Fachkräfte in Kitas wie Grundschullehrfachkräfte bezahlt werden müssen.

Bernhard Eibeck erläutert aus der Perspektive der GEW-Spitze die schwierige Ausgangslage des Tarifkonflikts mit seiner 25jährigen Geschichte im Übergang von BAT zu TVöD und fasst die wichtigsten Ergebnisse zusammen.

Das Interview mit einer Gruppenerzieherin, dem Leiter und der stellvertretenden Leiterin einer städtischen Kita mit 170 Kindern spiegelt die wesentlich erhöhten Anforderungen an den Job wider und zeigt, wie viel Ungereimtheiten, bezogen auf die ursprüngliche Zehn-Prozent-Forderung, die Streiktaktik und die Vermittlung der Verhandlungsergebnisse im Dickicht des Tarifgestrüpps entstanden und noch nicht geklärt sind. Die Unsicherheit über die Anerkennung von Dienstjahren nach Arbeitgeberwechsel ist dabei nur ein kleines Beispiel.

Die Folgen des Arbeitskampfes für das Jugendamt einer armen Kommunalverwaltung mit extrem defizitärem Haushalt und 80 Prozent (noch) nicht betroffenen freien Kita-Trägern erläutert Annette Berg, ehemalige Erzieherin und heute Leiterin des Jugendamtes der Stadt Essen.

Die beim Streik neu entwickelte Mobilisierung und Solidarität des Kita-Personals und der Sozialarbeiter stehen im Mittelpunkt abschließender Einschätzungen von vier ver.di-Aktiven aus derselben Kommune. Abschließend verbindet der ver.di-Insider Clemens Walter seine kritische Analyse der Stärken und Schwächen des Arbeitskampfes in der Öffentlichkeit mit den dabei deutlich gewordenen grundsätzlichen Fragen zur Tarifpolitik und neuen Mobilisierungsformen für den Sozial- und Erziehungsdienst im Vergleich zu anderen TVöD-Beschäftigten.

1. http://www.deutscher-kitaleitungskongress.de/images/downloads/BeWAK_Studie_2015.pdf; http://buendnis-jugendhilfe.de/2015/08/20/wissenschaftler-fuer-diegesellschaftliche-aufwertung-der-sozial-und-erziehungsberufe)/

2. ca. 80 Prozent der gewerkschaftlich organisierten ErzieherInnen und SozialarbeiterInnen sind ver.di-Mitglied, die übrigen 20 Prozent in der Bildungsgewerkschaft GEW 